\title{
Design and Analysis of Balloon-expandable Stainless Steel Coronary Stent
}

Pan Xiaolin, Shi Gengqiang, Li Yuan, Wu Shanshan, Chen Long

University of Shanghai for Science and Technology, Shanghai City, China

Abstract: This thesis uses "Volume Control" ballon-stent model, and according to results of deformation of a stent and vascular during implantation, we obtain the stress contours of the stent during implantation. The results conclude by ANSYS simulation show that during the implantation process, expansion of balloon body will make the narrow part of the blood wall fragment tough. After placement of the stent, due to blood flow in vessel makes that the inner wall of blood vessels has uneven distribution of shear stress; And, in the vicinity of the stent structure, it has a lower ${ }^{[7]}$ shear stress to vessel wall. During the expansion of the stent, the maximum principal stress concentrate on the connection between coronary stent itself and stiffeners.

Keywords: intravascular stent; balloon-stent model; finite element analysis; pressure-diameter curve

\section{Introduction}

The related symptoms of acute or chronic vascular obstruction have a great threat to human life; collected data show that blocking of heart head blood-vessel has become a main disease for threatening human health; in the mid-20th century, interventional operation technique started by application in therapeutic areas; the interventional operation technique is a minimally invasive technique and characterized with slightly injury, safety, and clinical efficiency etc. Therefore, the interventional operation technique attracts more and more attention by clinical surgeons and researchers; and in current therapy of vascular obstruction disease, intravascular stent intervention surgery has already become a main method; although stent generation supplements therapy method, and the therapy method has a long history from development up to mow; merely, we still have larger difficulty by adopting common method and technique to research stent performance; stent design and production is a complicated project with huge cost; in research and development, if using physical mechanical for carrying out experiment, the cost will burden heavier. In addition, stent size structure is compact, such as diameter of coronary stent is about $1.45 \mathrm{~mm}$, and wall thickness is less than $0.15 \mathrm{~mm}^{[6]}$; stent performance can not be detected by common detecting techniques; at the same time, some of parameters can not be obtained by current detection technique monitoring; and parameters play an important part for stent mechanical performance research; moreover, at present stent market has a strong competition, more and more novel stents with excellent property appear on market; therefore, intravascular stent research staff need to face bigger and bigger challenge and improve their stent design standard to make fast optimization for stent performance, which is their urgent

Copyright (C) 2017 Pan XL et al.

doi: $10.18686 /$ fm.v6.91

This is an Open Access article distributed under the terms of the Creative Commons Attribution-Non Commercial 4.0 International License (http://creativecommons.org/ licenses/by-nc/4.0/), permitting all non-commercial use, distribution, and reproduction in any medium, provided the original work is properly cited. 
affairs.

Advanced optimization technique could help research and development staff designs more and more high quality stent products; finite element analysis could meet the requirement in the aspect. Leading stent solid model in finite element analysis software and carrying out numerical modeling, and then stent performance parameters are obtained; according to the software feedback parameters we can make a structure optimization design to the stent; in this way, existing conception can be verified; reducing actual usage of stent object, which can not only lower research and development cost, but also can save time, and can speed up products research and development cycle.

\section{Application of finite element analysis in the intravascular stent}

In the related theory of research of the character of coronary stent expansion, Brauer ${ }^{[1]}$ and other scholars carry out different type stent external balloon expansion experiments; through the experiments, treatment effects of different materials and different design stent are analyzed; and the relation between balloon pressure and variable quantity of stent diameter has no great difference; generally there are five steps; the specific figure 1-1(a) is for your reference.

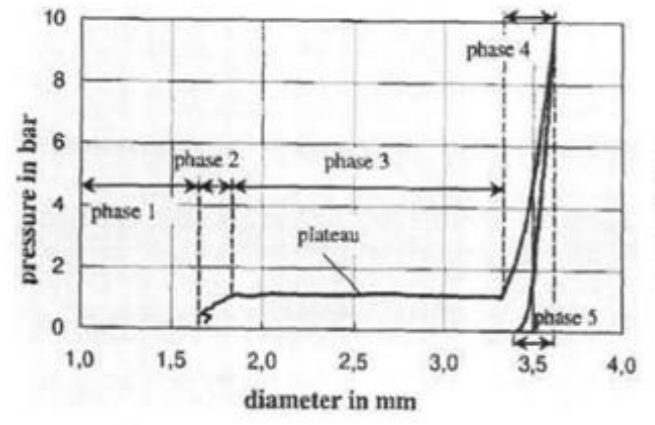

(a)

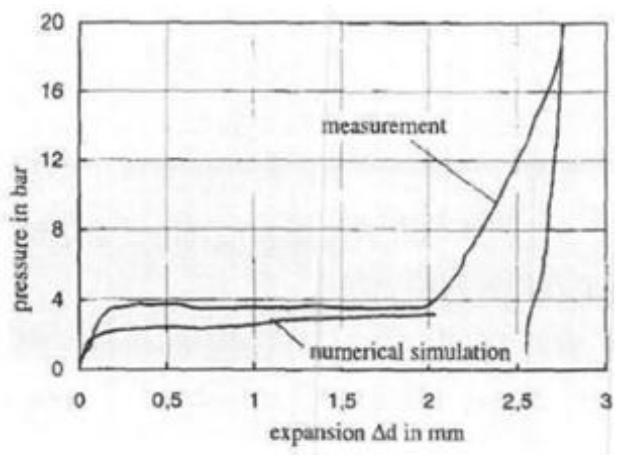

(b)

Figure 1-1 P-D Curve in Stent Expansion

From the figure, we know that intensive pressure-diameter curve (hereafter referred to as P-D curve) has pressure platform area which other curves hasn't; the stent expansion process can be regarded as height non-linear process. Foreign researchers ${ }^{[1]}$ build stent balloon model according to experiment conclusion; and after completion of building, stent expansion process is simulated, and P-D curve is obtained; as shown in the figure 1-1(b), From the figure, Brauer ${ }^{[1]}$ model covers pressure platform; and in the process of stent expansion simulation, part body is mainly simulated, not the main body.

In the following research, simulation in the stent expansion process attracts more and more attention in research; Dumoulin $^{[1]}$ and other researchers build different stent expansion test models, and all the models have a common feature(without regard to balloon model, that is, applying relevant pressure in the inner surface of stent model, to realize simulation in expansion process; then, the researchers combine stent model with vascular model to carry out research on which is mainly analyzing stent performance more deeply as well as influence of design method in clinical treatment, and comprehensive simulation to stent working environment as well as performance expression. In 1999, for the first time, by means of balloon-cascular model, Rogers ${ }^{[3]}$ and other scholars made a specific analysis for influence of vascular in all sorts of stent in balloon expansion process; the related research results show that corrugation type stent 
strut can reduce damage of vascular to the greatest extent. In recent years, Takashima ${ }^{[4]}$ and other researchers carry out calculation through stress of which vascular stent balloon model to the stent which is placed in the vessel, and at the same time, make an external experiment for verifying obtained results.

\section{Balloon Stent Finite Element Model Building}

\subsection{Stent Model Option and Build}

Aiming at the stent balloon in expansion process to make a research, which has an important significance for further research of stent performance. In current study, common balloon expansion stent finite element modeling has two kinds: one is directly pressing in the inner wall of the stent model to make an expansion study; and another is to build a stent balloon model, and then using balloon expansion to realize simulation in expansion process; obviously, the second model conforms to practical situation in the stent expansion; and more accurately complete deformation parameters can be obtained. However, in practical experiment, balloon must be folded and then can be used; in expansion, the effects of relationship between balloon and stent is more complex; In the research of Wang Weiqiang ${ }^{[5]}$ and Wu Wei ${ }^{[6]}$, data in the stent overall expansion has not been obtained through experiment; and the model has not been verified by experiment; if the balloon has crucial influence to stent expansion; then $\mathrm{Wu} \mathrm{Wei}^{\left[{ }^{[6]}\right.}$ scholar builds a balloon model which can control volume and has more reference meaning; Wu Wei ${ }^{[6]}$ designs a rhombus stent model to simulate expansion process, and makes analysis to stent performance.

\subsection{Balloon and vascular modeling and preliminary treatment}

\subsubsection{Original modeling}

Three dimensional CAD Solid works is adopted to build the related stent model, and then leading the model in the analyzing systems--ANSYS, to realize the finite element analysis. In the rhombus stent model, OD (outer diameter) $=5.92, \mathrm{~L}($ Length $)=20.4, \mathrm{~T}($ Thickness $)=0.1 \mathrm{~mm}$.

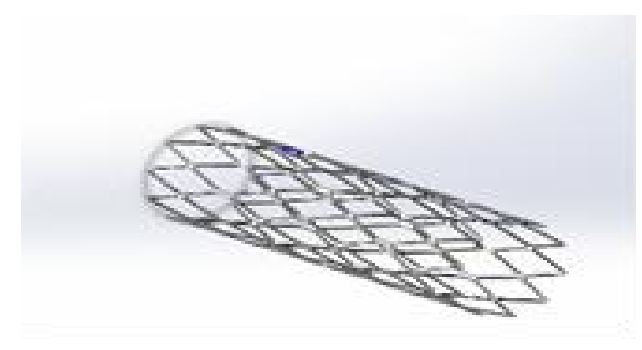

Figure 2-2 Stent external view

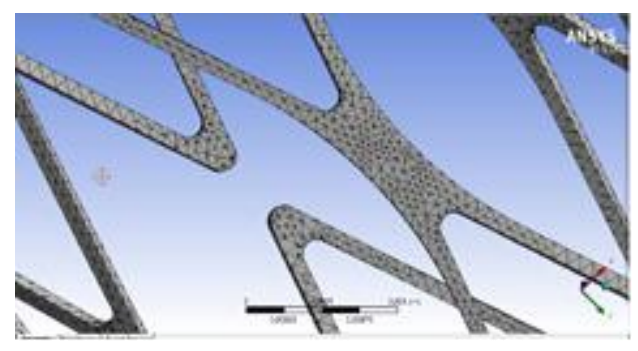

Figure 2-3 The stent after grid

Materials property: material is $316 \mathrm{~L}$ stainless steel. The model parameter is as following: tensile strength $=745 \mathrm{MPa}$, elasticity modulus $=200$; yield strength $=285$, Poisson's number $=0.32$, the yield platform slope $=1145 \mathrm{MPa}$. Due to the stent has symmetry characteristic, ratio of stent model and whole stent is 1:32, and ratio of circumference and the whole stent is $1: 16$, ratio of axial and the whole stent is $1: 2$.

\subsubsection{Balloon model}

Before building a balloon model, we need have a visual understand for practical balloon deformation; we can know it by watching real deformation process. Choosing length is $12 \mathrm{~mm}$ QMAV balloon to make a deformation experiment; 
experiment process is as following: connecting balloon to PTCA force pump, after that operating a pump and pressing balloon. Practical balloon expansion process is as the following figure 2-3; diameter of balloon in the beginning of pressing has a bigger change, and which shows quickly increasing; but when unfolded balloon after pressing is filled with liquid gradually, diameter length hasn't obverse change by continually pressing. The reason lies in balloon volume limit after the balloon being filled by liquid; using a vertical cylindrical can build a balloon model, which is shown as figure 2-4: diameter and length are 1.35 and 12.5 respectively, which is conform to setting conditions. According to feature of stent model, the ratio of designed stent volume and whole stent volume is 1:32. The balloon model material chooses virtual elastic material; and setting poisson's ratio is 0.500 , and modulus of elasticity is $13 \mathrm{MPa}$. Built balloon models are divided grid which generates a finite element model including a node and a unit, Adopting shell181 to operate, and setting the shell unit thickness is $0.46 \mathrm{~mm}$.

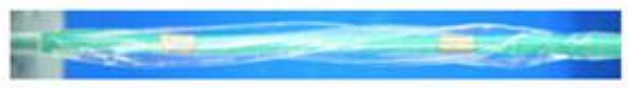

(a)

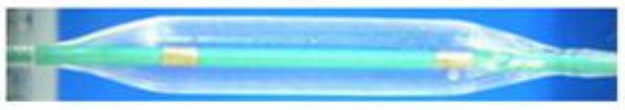

(c)

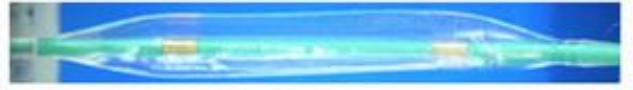

(b)

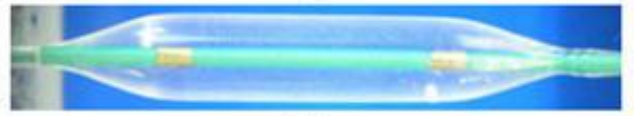

(d)

Figure 2-3 The Balloon Expansion Process

(a) Folded balloon; (b)starting Pressing The Balloon; (c)The Balloon Stress is $0.05 \mathrm{MPa}$; (d) Stress is $1.5 \mathrm{MPa}$

In order to control balloon volume in the practical expansion process, a controlling rigid body is added; shown as figure 2-4: that is, after balloon filled with liquid, pressure is rising, which generates a tiny balloon deformation. By adjusting rigid body diameter, the maximum expansion diameter of the balloon can be controlled indirectly. In the experiment, a stent in the text, make sure $\mathrm{D}$ is $3.37 \mathrm{~mm}$ which is the same as balloon deformation in $1.2 \mathrm{MPa}$.

\subsubsection{Stent and balloon as well as vascular combined model}

As shown in the figure 2-7, completing the stent and the balloon as well as vascular the whole assembly; the ballon and rigid body align to the stent, to make the stent and the balloon parallel and symmetrical to each other; building a contact element between the inner surface of the stent and the balloon, and generating rubbing action is smaller, which can be neglected.

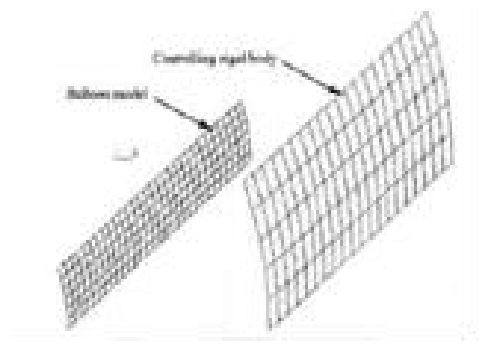

Figure 2-4(a) Balloon and Volume Control Rigid;

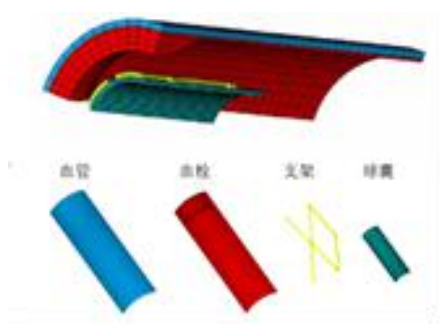

Figure 2-4(b) stent and balloon and vascular combined model 


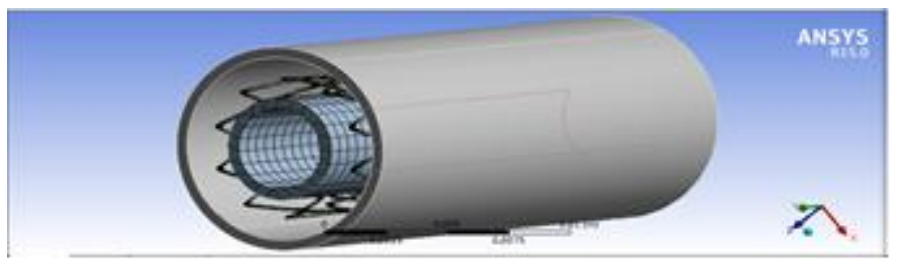

Figure 2-4(c) stent and balloon and vascular combined model

\subsection{Solution Controls}

In balloon stent model, applying different pressure, and making a record of balloon deformation in different pressure, and then counting radial reply data, axial response data and axial shortening data, as following:

Radial reply data=the biggest pressure D-After pressure unloading D

The biggest pressure

Axial response data $=$ the biggest pressure L-After pressure unloading $\mathrm{D}$

The biggest pressure $\mathrm{L}$

Axial shortening data $=\underline{\mathrm{L}-\text { After the pressure unloading } \mathrm{L}}$

$\mathrm{L}$

Recording change relation between pressure load $\mathrm{P}$ and diameter $\mathrm{D}$, and drawing a P-D curve; at the same time, paying attention to variation of stresses in the process of stent expansion deformation, especially for the distribution of the maximum stress.

\subsection{Finite Element Analysis and Experiment Results}

\subsubsection{Entire deformation}

In figure 2-5, the stent is completely deformation, and (a1-a4) is a specific result after stent-balloon model (the results contain control rigid body); (b1-b4) shows that relevant $\mathrm{Wu}$ Wei ${ }^{[6]}$ in experiment, the practical stent in the same pressure corresponding results. In figure 2-1, stent deformation parameters are made inductive integration; from the results, experiment results conform to simulation results; through research of combing stent with balloon combined model, we know that stent deformation parameters is closer to stent expansion data.

\begin{tabular}{ccc}
\hline & $\begin{array}{c}\text { The simulation results of this } \\
\text { experiment }\end{array}$ & $\begin{array}{c}\text { Wu Wei's experimental } \\
\text { results }\end{array}$ \\
\hline Maximum loading diameter /mm & 3.76 & $3.76 \pm 0.01$ \\
Radial recovery $\%$ & 5.66 & $5.65 \pm 0.09$ \\
Axial recovery /\% & -1.04 & $-1.06 \pm 0.12$ \\
Axial shortening /\% & 4.11 & $4.11 \pm 0.08$ \\
\hline
\end{tabular}




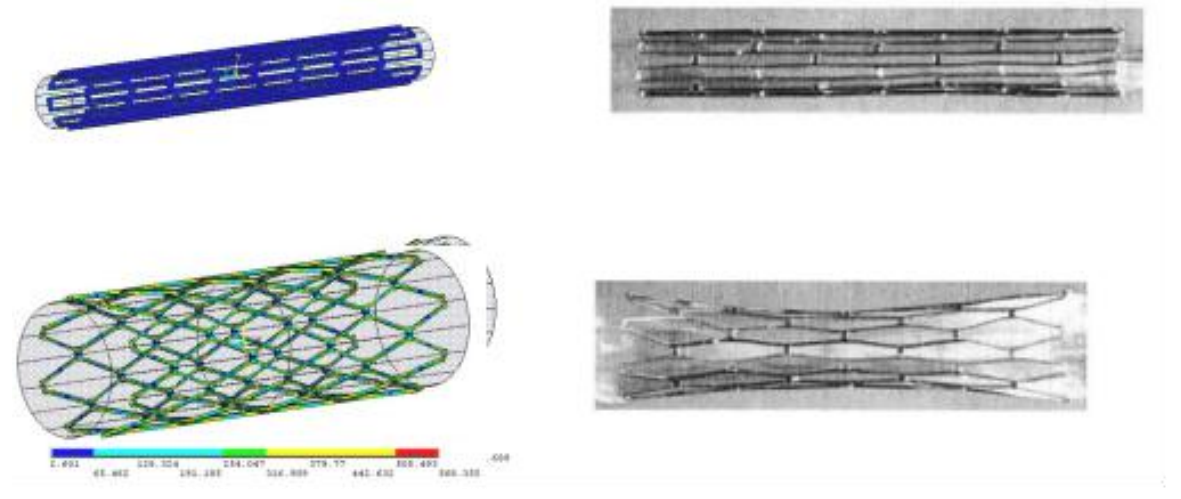

(a1) Stent-balloon model on 0MPa; (b1) under the same pressure of picture

(a2) Stent-balloon model on $0.1 \mathrm{MPa}$; (b2) $0.1 \mathrm{MPa}$ under the pressure of picture
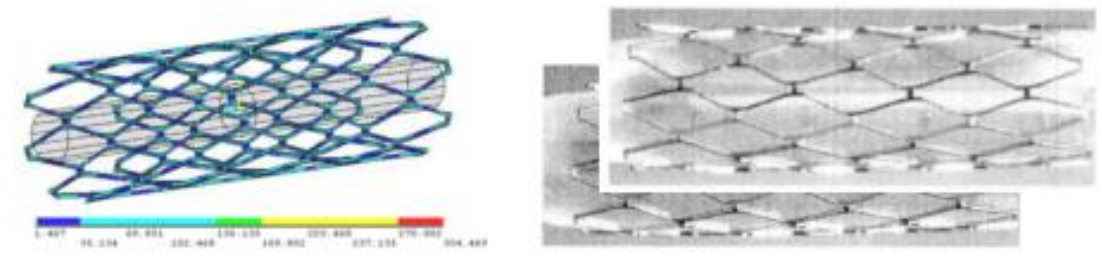

(a3) Stent-balloon model on 1.2MPa; (b3) 1.2Mpa under the pressure of picture

(a4) After the removal of the balloon pressure state; (b4) the picture of removing pressure

At $0 \mathrm{Mpa}$, the balloon is in an expansion of state, and each part has no deformation, and there is no residual stress in junction. Dogbone phenomenon is connected with balloon pressure, and when pressure of balloon model reach to $0.10 \mathrm{MPa}$, stent model will appear the phenomenon; and this typical phenomenon is also verified in Wu Wei's experiment pictures, when pressure in balloon is $1.20 \mathrm{MPa}$, the balloon model is restrained by controlling rigid body to reach balance diameter maximum value; at the same time, in the stent model, diameter of each place are same, and when diameter is in pressure, balloon expansion reach the limit, and stent diameter is reach maximum value. After the pressure being removed, stent model will appear elasticity, and diameter will begin to shrink gradually, simultaneously, residual stress will appear, and the results are accordance with Wu Wei experiment results.

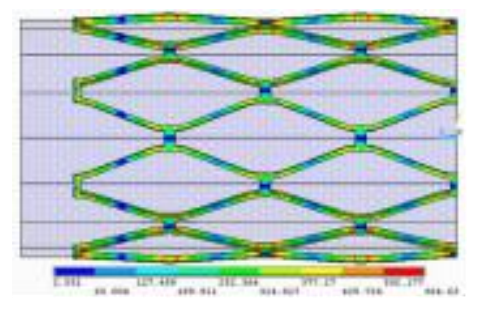

\subsubsection{P-D Curve}


In simulation experiment, the P-D curve is obtained, which could be referred to figure 2-6. From the figure, we know that the applying pressure is $0-0.1 \mathrm{MPa}$ at the beginning of expansion; the model Dc increases smoothly and stent Dc range ability is small. When balloon pressure is $0.1 \mathrm{MPa}$; from both simulation results and experiment results, we can find the pressure platform is changed obviously; when the balloon pressure is on stent Dc $3.15 \mathrm{~mm}$, speed increases, and when model pressure Dc value is $3.56 \mathrm{~mm}$, speed increases obviously. Model and stent applying pressure is $1.2 \mathrm{MPa}$, Dc Value is $3.76 \mathrm{~mm}$, and then removing the pressure, both appear radial reply, and both Dc value also recovers $3.55 \mathrm{~mm}$.

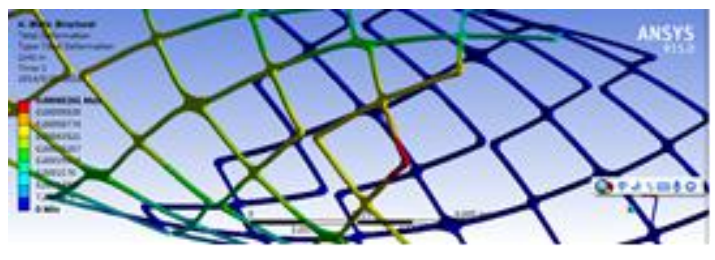

Figure 2-6 P-D Curves

\subsubsection{Local plastic deformation and the maximum stress distribution}

When balloon expansion to the maximum diameter, stent model in the joint between major structure and reinforcing rib will come up plastic deformation, and the maximum stress also distributes in the joint position; the plastic deformation in the joint and the maximum stress diagram shown in figure 2-7, 2-8.

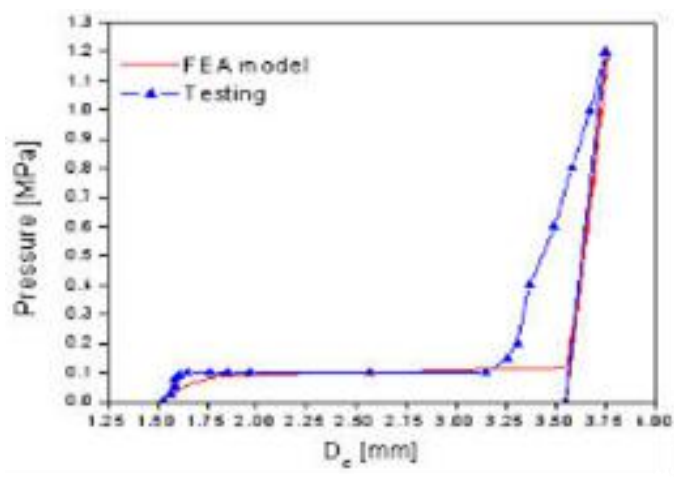

The figure 2-7: The plastic deformation in the joint

The figure 2-8: The maximum stress distribution

\section{Result and Analysis}

From the figure 2-4, the simulation of balloon expansion deformation generates obvious effect through volume control method to build a balloon-stent. In the building model, in the circumstance of applying the maximum pressure in the balloon, diameter of model and stent balloon are same, so the author mentioned the building stent-balloon can simulate the whole process of stent expansion with higher accuracy, at the same time, we can obtain more accurate relevant experiment data for further research in convenience.

Moreover, according to different diameter after the stent expansion, we adjust model parameters, and then we can realize to control the position of rigid body in the balloon; and the maximum expansion diameter of model can be obtained, and then we can carry out more accurate simulation to each expansion process. 
Finite element analysis can also estimate to the maximum stress area in qualitative change of deformation. In novel stent design, the maximum stress position can be found during expansion by the finite element analysis; and contrasting with strength of extension of stent materials, we can avoid the stent to be damaged on account of expansion; on this basis, we can make an instruction for stent design to reduce stent stress, therefore the stent can not be damaged in expansion, and conform to clinical application standard.

This thesis adopts Solid works to build a model including a stent, a balloon and vascular and make an analysis to the stent in vascular; we can obtain shear stress and deformation parameters of rhombus stent in vascular, therefore we can make a systematic evaluation for the rhombus stent. The computer simulation obtained results prove that balloon body expansion will damage the inner wall of vascular in process of stent placing in the vascular, which will make the inner wall of vascular unsmooth. After placement of the stent in the vascular, blood flowing will lead in shear force distribution unbalance; at the same time, closed shear force is smaller in stent surrounding. During stent expansion, the maximum stress concentrates on the joint of the coronary stent body and reinforcing rib, and the maximum plastic deformation also appears in the joint; therefore, the structure optimization of joint of body and stiffeners should be paid attention in stent design process.

\section{Reference}

1. Brauer H, Stolpmann J, Hallman H et al. Measurement and numerical simulation of the dilatation behavior of coronary stents. Materialwissenschaft und Werkstofftechnik, 1999, 30(12):876-885.

2. Dumoulin C, Cochelin B. Mechanical behaviour modelling of balloon-expandable stems. Journal of Biomechanics, 2000, 33:1461-1470.

3. Rogers C, Tseng D Y, Squire J C et al. Balloon-Artery Interactions During Stent Placement: A Finite Element Analysis Approach to Pressure, Compliance, and Stent Design as Contributors to Vascular Injury. Circulation Research, 1999, 84:378-383.

4. Takashima K, Kitou T, Mori K et al. Simulation and experimental observation of contact conditions between stents and artery models. Medical Engineering \& Physics, 2007,29(3):326-335.

5. Wang Weiqiang. Yang Dazhi, Qi-Min, Finite Element Analysis of Coranary Stent Anti Compression Property 2006, 23(5): 1008-1012. [J] Bio-medical Engineering Journal.

6. Wu Wei, Finite Element Analysis and Structure Topological optimization of Human Body Intravascular stent [D][D] Dalian University of Technology, 2007.1-150.

7. Li Hongxia, Wang Xicheng, Hemodynamics in The Narrow Vascular Implemented Stent and Simulation of Action of Bloodstream to Vascular Wall[C]// Chinese Society of Theoretical and Applied Mechanics 2009Abstract of thesis.2009.

8. Ge Junbo, Liu Xuebo, Recognition of Drug Eluting Stent and Restenosis Machine-made. China Angiocardiopathy Journal 2006, 33(8):681-683.

9. Experimental medicine and its initial applications in man. Acta Medica Scandinavica. Supplementum 1975, 579:3-32.

10. Seldinger. Catheler replacement of the needle in percutan $\omega$ us arteriography; a new technique.

11. Serruys P W, transluminal angioplasty. New England Journal of Medicine, 1987,316: 701-706.

12. Zhao Zhenxin, Liu Daozhi, Sun Kang etc. Nitinol Introvascular Stent Finite Element Analysis and Fatigue Test [J][J]. China Medical Apparatus and Instruments, 2008, 32(5): 373-376.

13. Chen Xidong, Yang Jie, Zhao Xiao-dong, Finite Element Analysis Development State and Application [J]. China Informatization of Manufacturing Industry; Academic Edition 2010 (6): 6-8.

14. Zhu Aiping, Chen Jian, Research Progress of Intravascular Stent. Biomedical Engineering Journal2010 (6): 6-8. 